Supporting Information

\title{
Spectroscopic and Electrical Characterizations of Low Damage Phosphorous-doped Graphene via Ion Implantation
}

Shih-Ming He ${ }^{\#}$, Cheng-Chun Huang ${ }^{\#}$, Jhe-Wei Liou ${ }^{\$}$, Wei-Yen Woon $\$$, Ching-Yuan

$$
\mathrm{Su}^{\dagger+* *}
$$

\# Graduate Institute of Energy Engineering, National Central University, Tao-Yuan 32001, Taiwan

† Dep. of Mechanical Engineering, National Central University, Tao-Yuan 32001,

Taiwan

\$Dep. of Physics, National Central University, National Central University, Taoyuan City 32001, Taiwan

To whom correspondence should be addressed: (C. Y. Su): cysu@ncu.edu.tw 
(a)

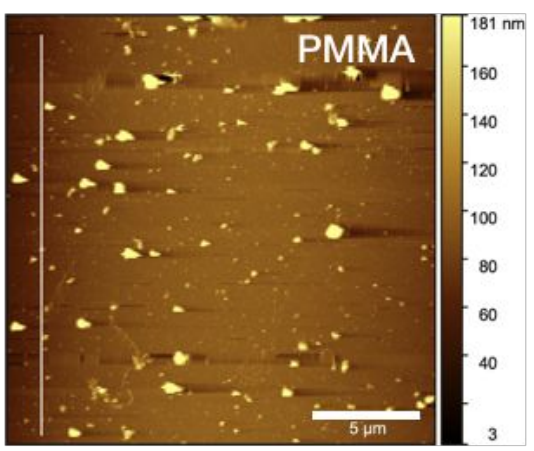

(c)

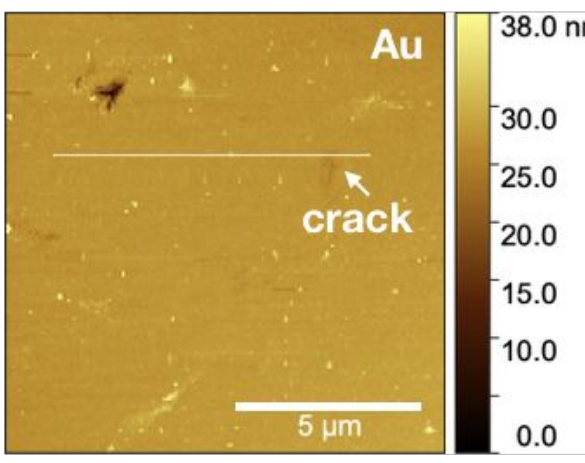

(b)

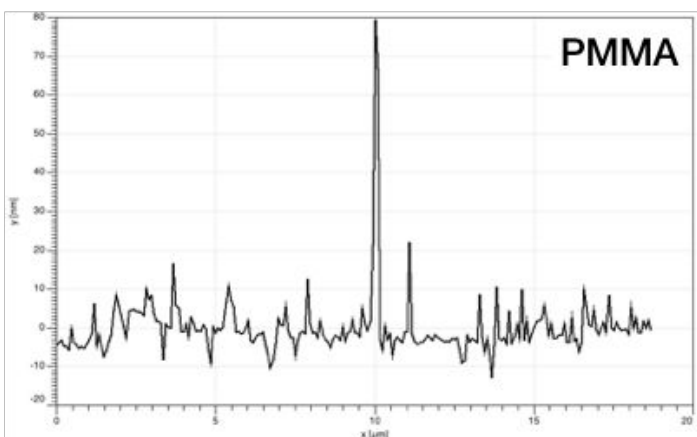

(d)

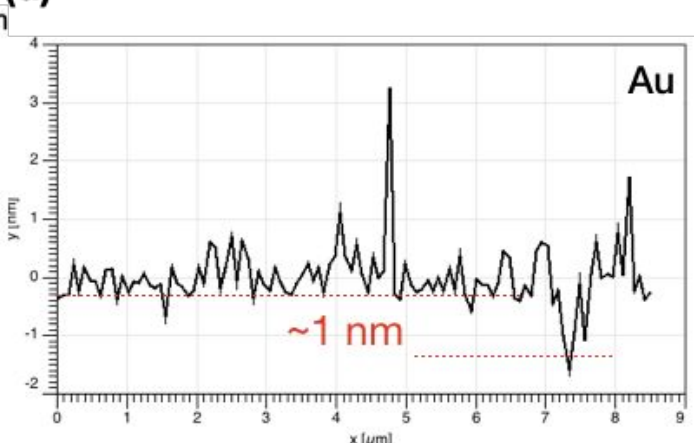

Figure S1. AFM images of representative monolayer graphene with different transfer methods: (a) Morphology and (b) cross-sectional analysis of typical PMMA transfer method, respectively. (c) Morphology and (d) cross-sectional analysis of Au-assisted transfer method, respectively.
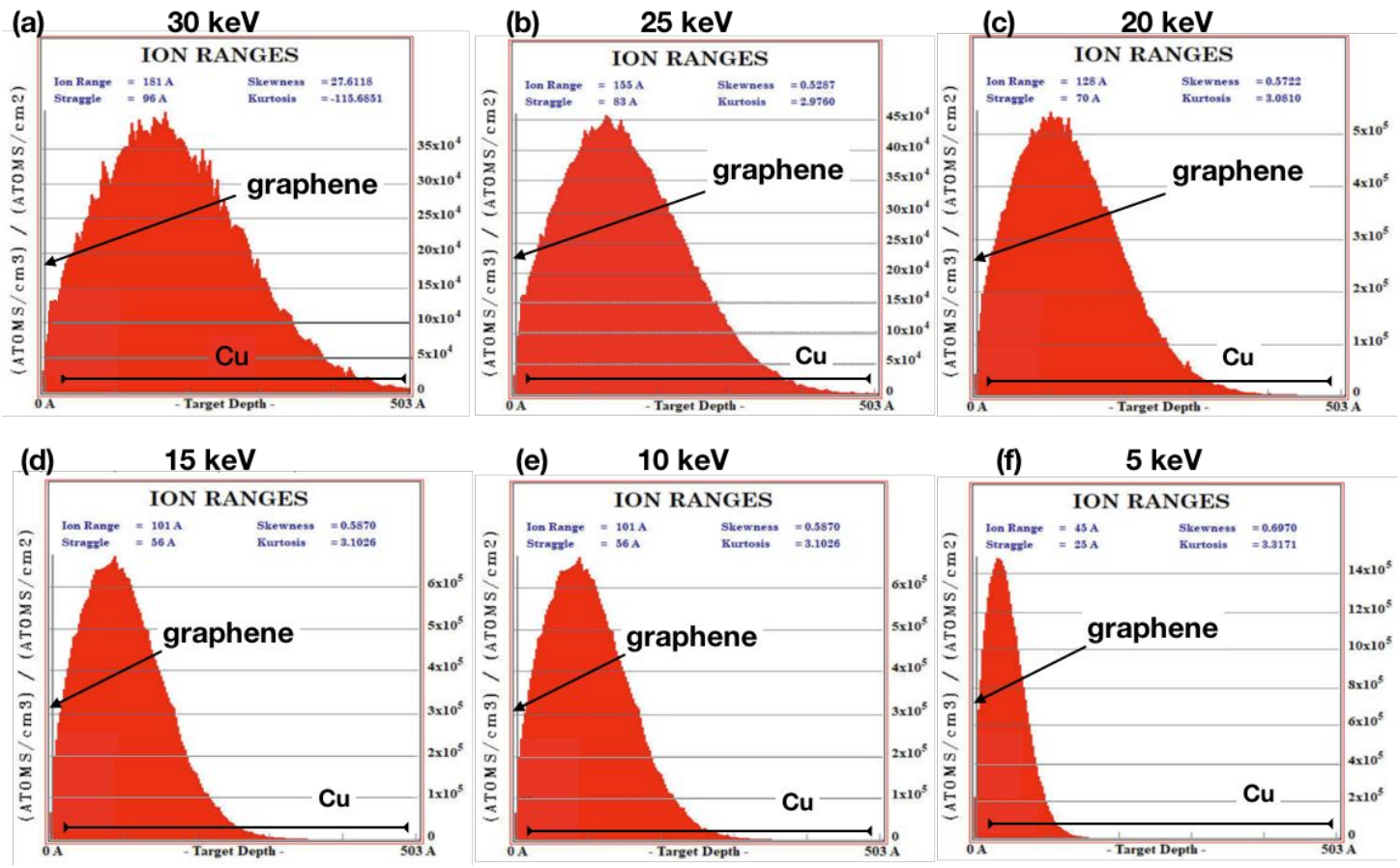

Figure S2. SRIM simulation of $\mathrm{P}$ ions penetrate into as-grown graphene with different energies of (a) $30 \mathrm{keV}$, (b) $25 \mathrm{keV}$, (c) $20 \mathrm{keV}$, (d) $15 \mathrm{keV}$, (e) $10 \mathrm{keV}$, (f) $5 \mathrm{keV}$. 
(a) $30 \mathrm{~nm} \mathrm{Au/graphene/Cu}$
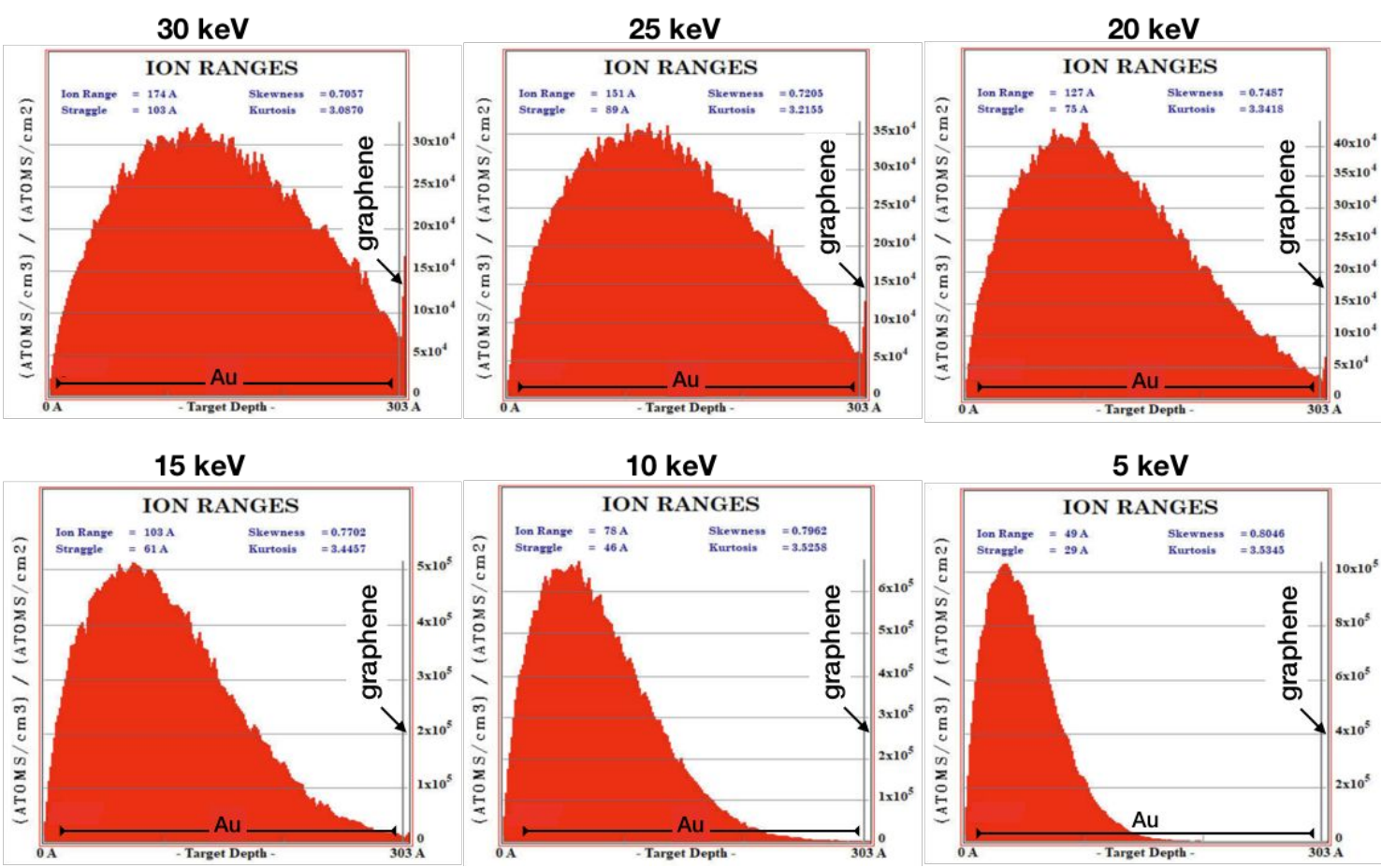

(b) $25 \mathrm{~nm}$ Au/graphene/Cu
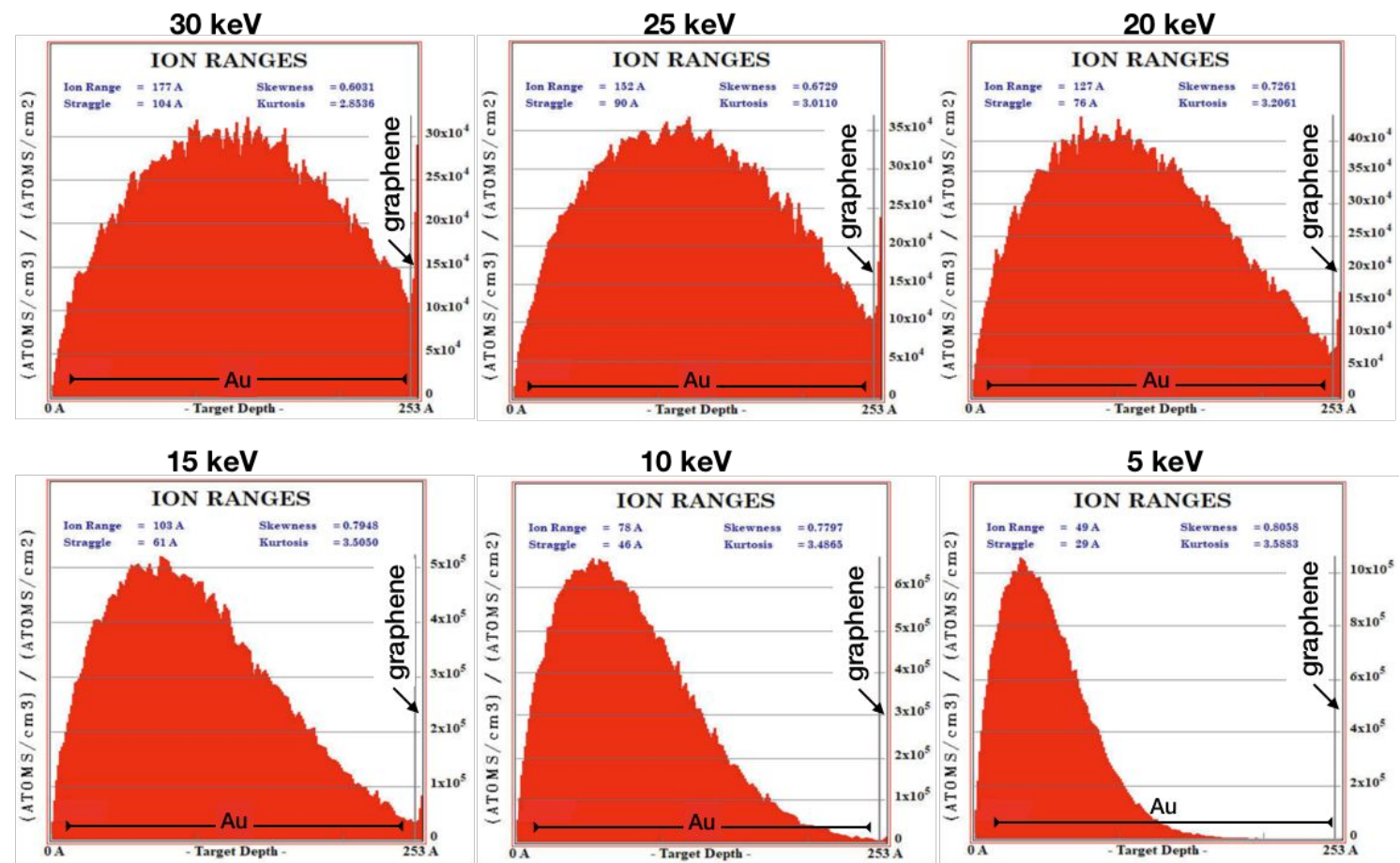
(c) $20 \mathrm{~nm} \mathrm{Au/graphene/Cu}$

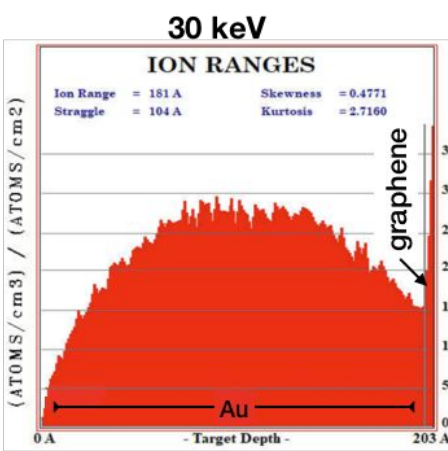

$15 \mathrm{keV}$

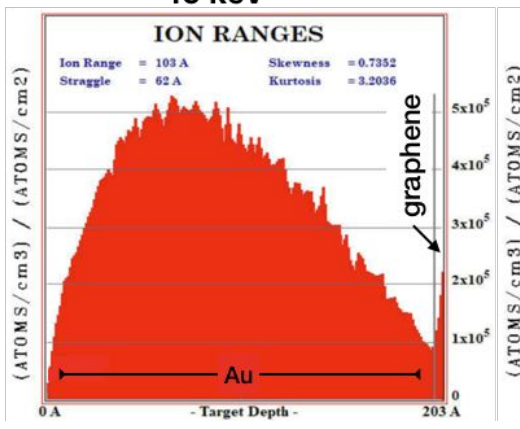

(d) $15 \mathrm{~nm} \mathrm{Au/graphene/Cu}$
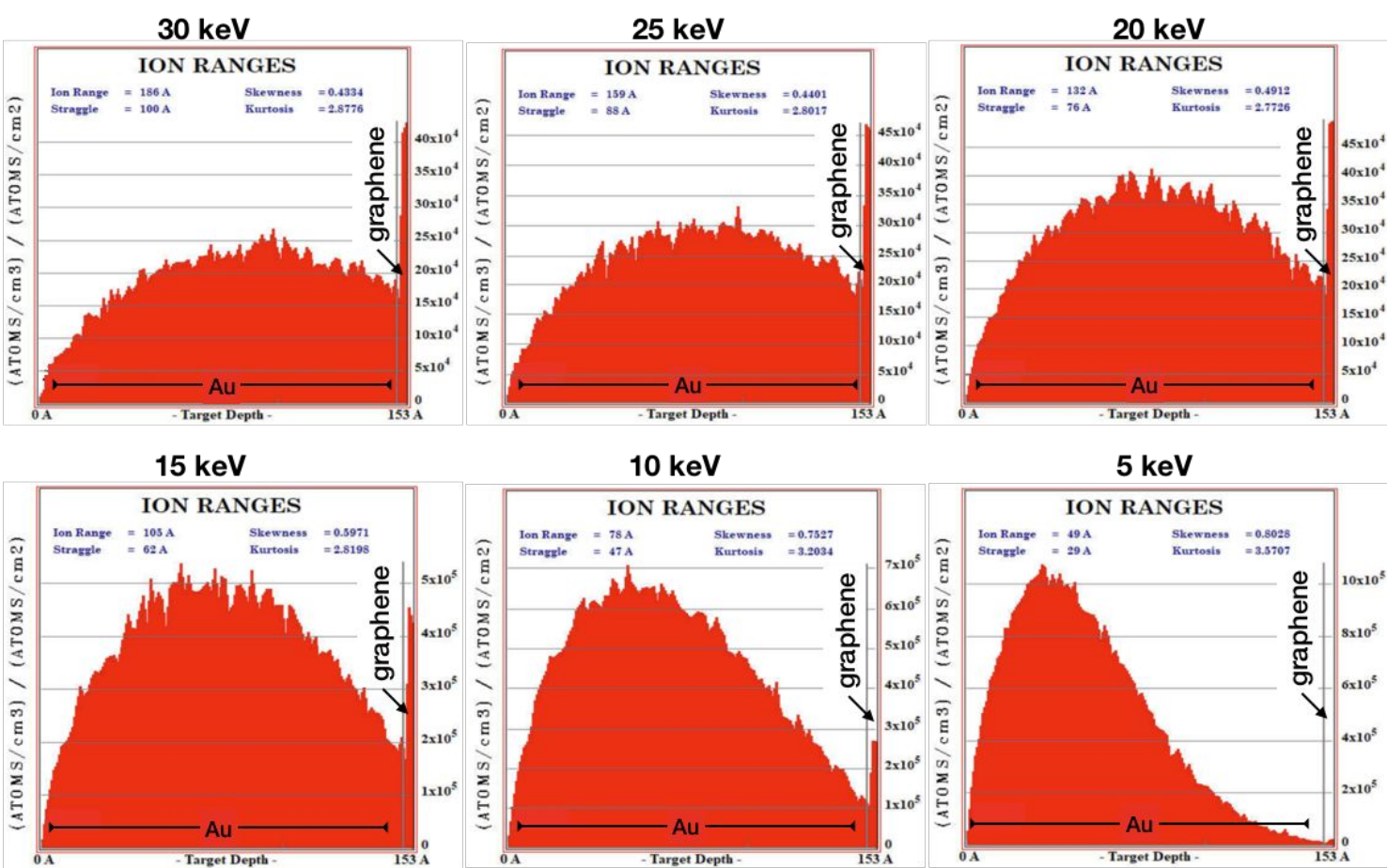

$5 \mathrm{keV}$

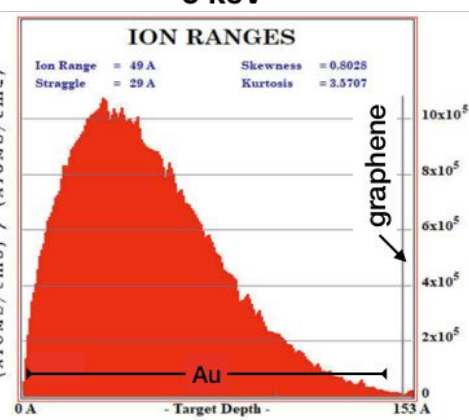


(e) $10 \mathrm{~nm} \mathrm{Au/graphene/Cu}$

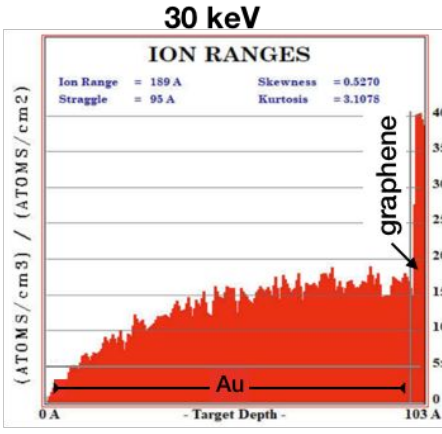

$15 \mathrm{keV}$

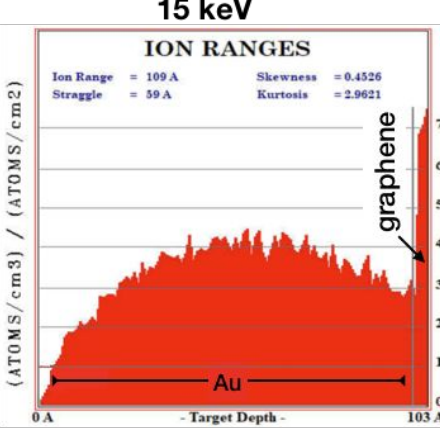

(f) $5 \mathrm{~nm} \mathrm{Au/graphene/Cu}$

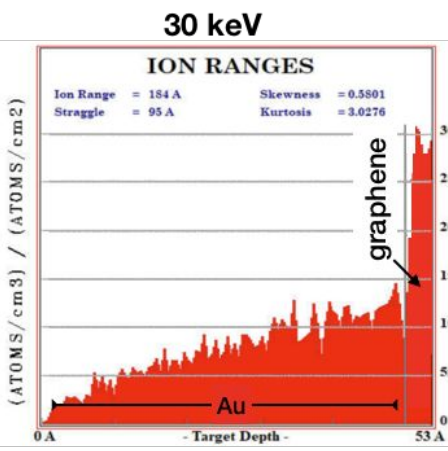

$15 \mathrm{keV}$

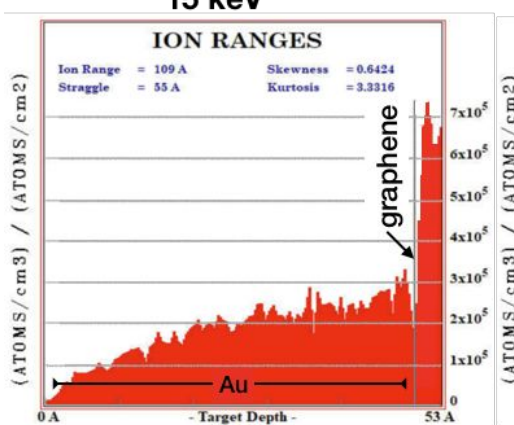

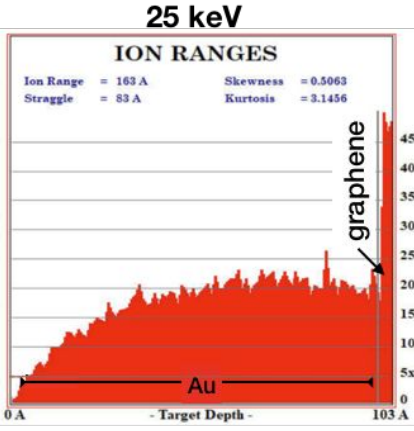

$10 \mathrm{keV}$

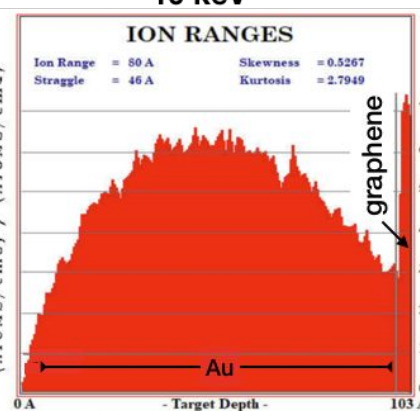

$20 \mathrm{keV}$

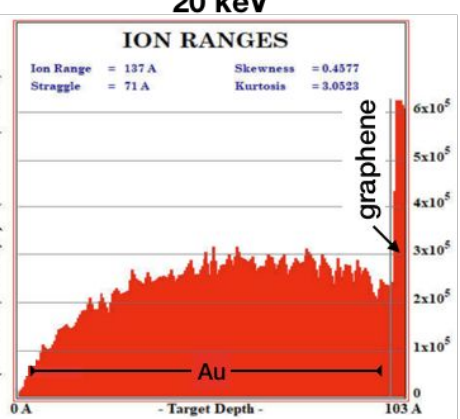

$5 \mathrm{keV}$

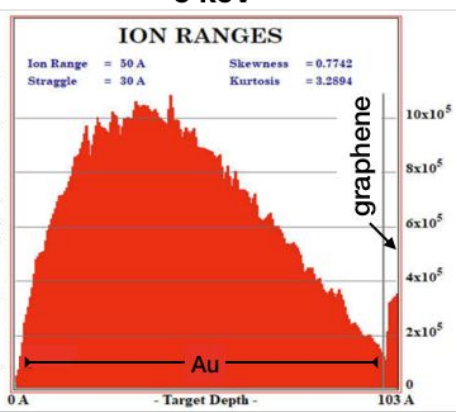

$25 \mathrm{keV}$

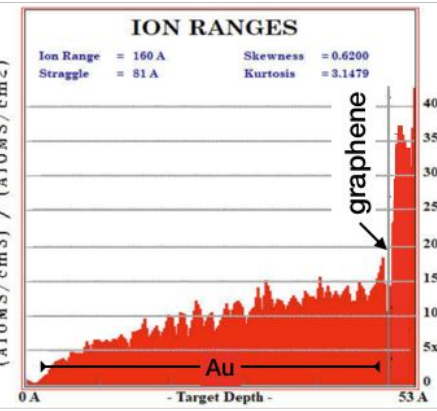

$10 \mathrm{keV}$

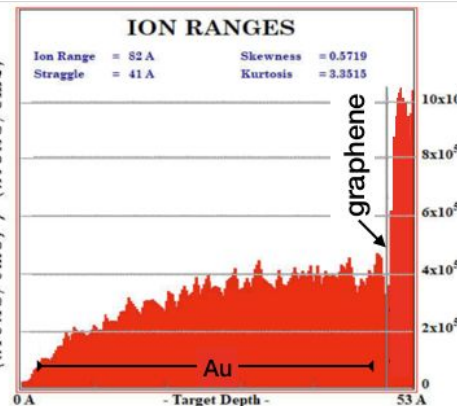

$20 \mathrm{keV}$

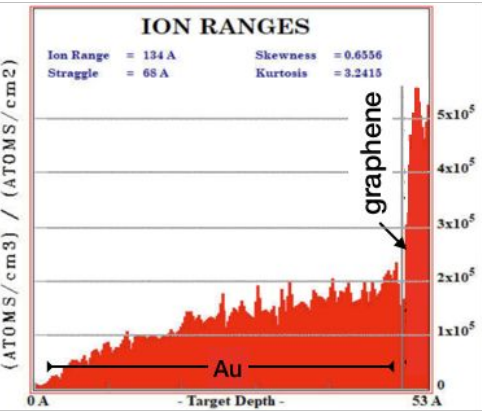

$5 \mathrm{keV}$

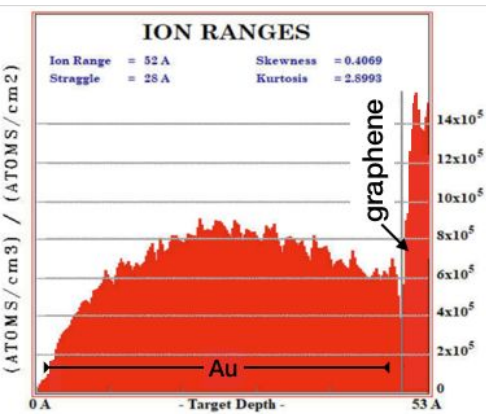

Figure S3. SRIM simulation of $\mathrm{P}$ ions penetrate into different thickness of designed structure with different energy. (a) $30 \mathrm{~nm} \mathrm{Au} / g r a p h e n e / \mathrm{Cu}$. (b) $25 \mathrm{~nm} \mathrm{Au} / \mathrm{graphene} / \mathrm{Cu}$. (c) $20 \mathrm{~nm} \mathrm{Au} /$ graphene/Cu. (d) $15 \mathrm{~nm} \mathrm{Au} /$ graphene/Cu. (e) $10 \mathrm{~nm} \mathrm{Au} /$ graphene/Cu. (f) 5 $\mathrm{nm} \mathrm{Au/graphene/Cu}$. 


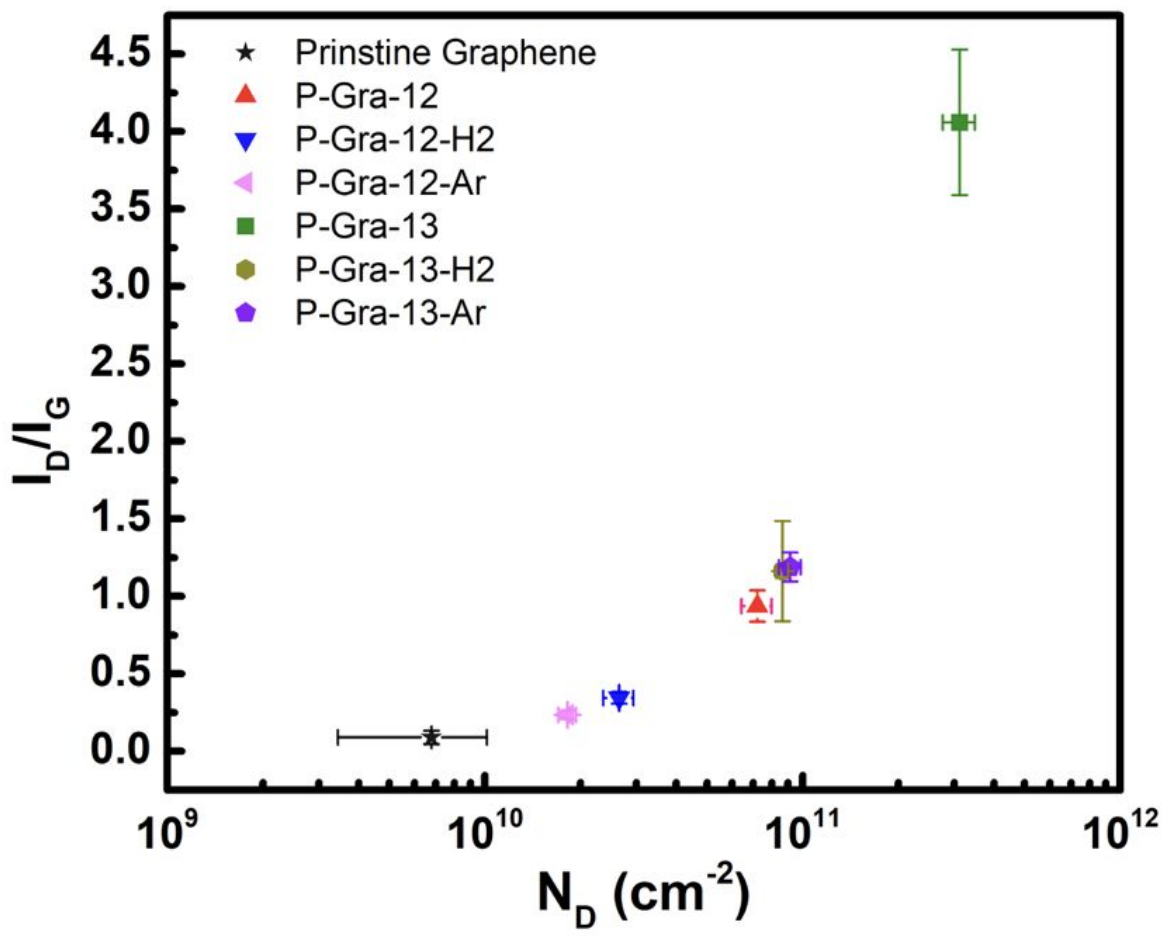

Figure S4. $\mathrm{I}_{\mathrm{D}} / \mathrm{I}_{\mathrm{G}}$ versus defect density obtained from pristine graphene and P-Gra
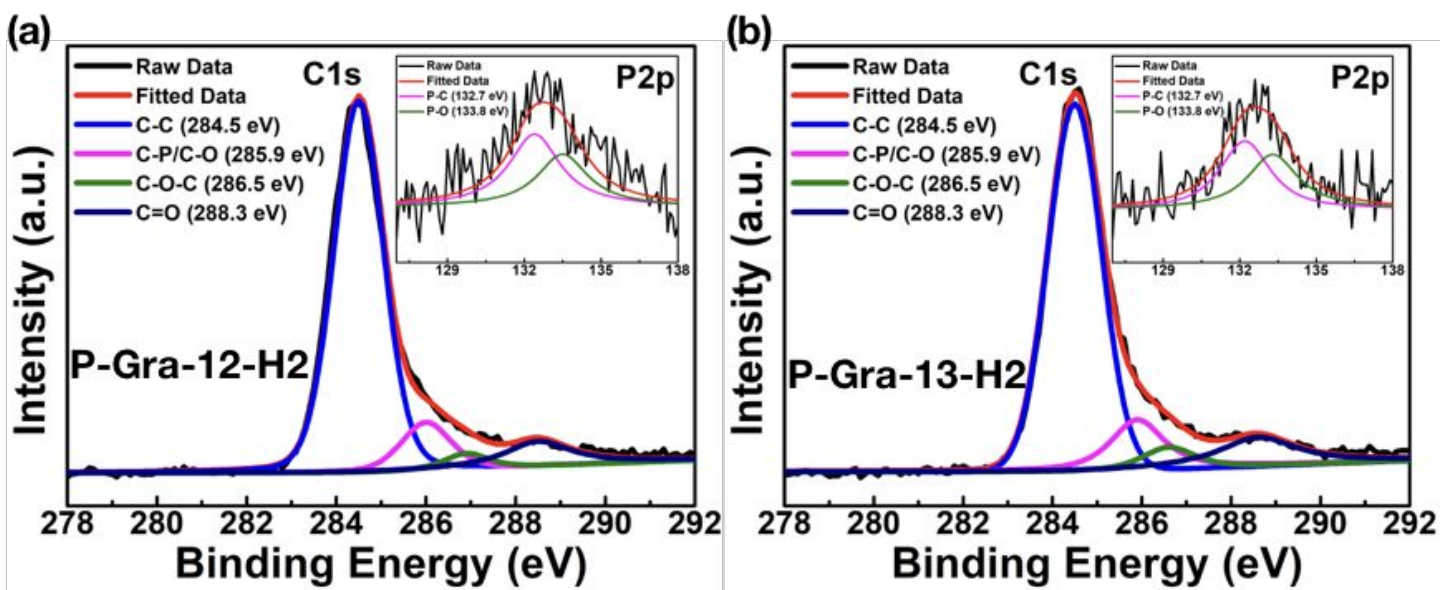

Figure S5. XPS analysis of C1s and P2p spectra for (a) P-Gra-12-H2. (b) P-Gra-13-H2. 


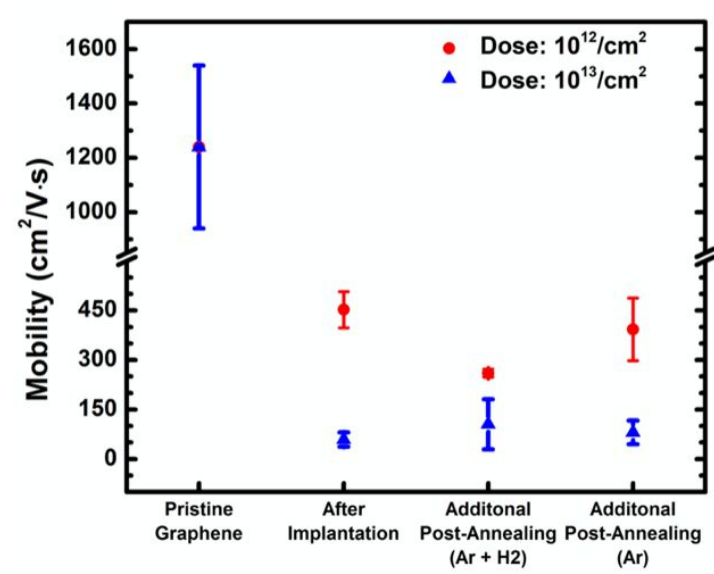

Figure S6. Statistical analysis of the mobility measured by Hall measurement for all samples.

Table S1. Comparison of different implant elements, energy, ion fluences and $\mathrm{I}_{\mathrm{D}} / \mathrm{I}_{\mathrm{G}}$

\begin{tabular}{|c|c|c|c|c|c|}
\hline Element & Energy & Target & $\mathbf{I}_{\mathbf{D}} / \mathbf{I}_{\mathbf{G}}$ & Ion fluence $\left(\mathrm{cm}^{-2}\right)$ & \\
\hline \multirow[t]{2}{*}{ B } & $35 \mathrm{eV}$ & $\mathrm{Gra} / \mathrm{SiO}_{2}$ & 0.55 & $10^{14}$ & 32 \\
\hline & & & 3.3 & $2 \times 10^{15}$ & \\
\hline \multirow[t]{2}{*}{$\mathrm{N}$} & $35 \mathrm{eV}$ & $\mathrm{Gra} / \mathrm{SiO}_{2}$ & 1.27 & $10^{14}$ & \\
\hline & $20 \mathrm{eV}$ & $\mathrm{Gra} / \mathrm{SiO}_{2}$ & $\alpha-C$ & $6 \times 10^{15}$ & \\
\hline \multirow[t]{2}{*}{$\mathrm{F}$} & $35 \mathrm{eV}$ & $\mathrm{Gra} / \mathrm{SiO}_{2}$ & 0.69 & $10^{14}$ & \\
\hline & & & 4.96 & $2 \times 10^{15}$ & \\
\hline \multirow[t]{4}{*}{$\mathrm{N}$} & $20 \mathrm{eV}$ & $\mathrm{MLG} / \mathrm{SiO}_{2}$ & 0.22 & $10^{14}$ & 53 \\
\hline & $30 \mathrm{eV}$ & & 1.25 & $10^{14}$ & \\
\hline & $1 \mathrm{keV}$ & & $\alpha-C$ & $10^{14}$ & \\
\hline & $20 \mathrm{eV}$ & $\mathrm{MLG} / \mathrm{SiO}_{2}$ & 1.27 & $10^{15}$ & \\
\hline \multirow[t]{7}{*}{$P$} & $20 \mathrm{keV}$ & $\begin{array}{l}\text { Pristine } \\
\text { Graphene }\end{array}$ & 0.12 & - & This work \\
\hline & & P-Gra-12 & 0.94 & $10^{12}$ & \\
\hline & & P-Gra-12-H2 & 0.34 & $10^{12}$ & \\
\hline & & $P-G r a-12-A r$ & 0.23 & $10^{12}$ & \\
\hline & & P-Gra-13 & 4.06 & $10^{13}$ & \\
\hline & & P-Gra-13-H2 & 1.16 & $10^{13}$ & \\
\hline & & P-Gra-13-Ar & 1.19 & $10^{13}$ & \\
\hline
\end{tabular}

$\alpha$-C: amorphous carbon; $M L G$ : multi-layer graphene 
Table S2. Concluded bonding structure on pristine graphene and various P-Gra samples.

\begin{tabular}{|c|c|c|c|c|c|c|}
\hline & C-C & C-O/C-P & C-O-C & C=O & P-C & P-O \\
\hline $\begin{array}{c}\text { Pristine } \\
\text { Graphene }\end{array}$ & $85.09 \%$ & $8.10 \%$ & $3.76 \%$ & $3.04 \%$ & - & - \\
\hline P-Gra-12 & $74.72 \%$ & $6.55 \%$ & $9.82 \%$ & $8.91 \%$ & $39.13 \%$ & $60.87 \%$ \\
\hline P-Gra-12-Ar & $72.51 \%$ & $13.97 \%$ & $5.22 \%$ & $8.30 \%$ & $65.25 \%$ & $34.75 \%$ \\
\hline P-Gra-12-H2 & $75.78 \%$ & $10.77 \%$ & $3.60 \%$ & $9.85 \%$ & $58.06 \%$ & $41.94 \%$ \\
\hline P-Gra-13 & $67.02 \%$ & $16.67 \%$ & $7.38 \%$ & $8.93 \%$ & $44.62 \%$ & $55.38 \%$ \\
\hline P-Gra-13-Ar & $71.97 \%$ & $11.18 \%$ & $7.62 \%$ & $9.23 \%$ & $58.23 \%$ & $41.77 \%$ \\
\hline P-Gra-13-H2 & $69.59 \%$ & $12.98 \%$ & $5.31 \%$ & $12.12 \%$ & $53.49 \%$ & $46.51 \%$ \\
\hline
\end{tabular}

